\title{
Unrecognised bipolar disorder in primary care patients with depression ${ }^{\dagger}$
}

\author{
Daniel J. Smith, Emily Griffiths, Mark Kelly, Kerry Hood, Nick Craddock and Sharon A. Simpson
}

\section{Background}

Bipolar disorder is complex and can be difficult to diagnose. It is often misdiagnosed as recurrent major depressive disorder.

\begin{abstract}
Aims
We had three main aims. To estimate the proportion of primary care patients with a working diagnosis of unipolar depression who satisfy DSM-IV criteria for bipolar disorder. To test two screening instruments for bipolar disorder (the Hypomania Checklist (HCL-32) and Bipolar Spectrum Diagnostic Scale (BSDS)) within a primary care sample. To assess whether individuals with major depressive disorder with subthreshold manic symptoms differ from those individuals with major depressive disorder but with no or little history of manic symptoms in terms of clinical course, psychosocial functioning and quality of life.
\end{abstract}

\section{Method}

Two-phase screening study in primary care.

\section{Results}

Three estimates of the prevalence of undiagnosed bipolar disorder were obtained: $21.6 \%, 9.6 \%$ and $3.3 \%$. The $\mathrm{HCL}-32$ and BSDS questionnaires had quite low positive predictive values (50.0 and $30.1 \%$ respectively). Participants with major depressive disorder and with a history of subthreshold manic symptoms differed from those participants with no or little history of manic symptoms on several clinical features and on measures of both psychosocial functioning and quality of life.

\section{Conclusions}

Between 3.3 and $21.6 \%$ of primary care patients with unipolar depression may have an undiagnosed bipolar disorder. The HCL-32 and BSDS screening questionnaires may be more useful for detecting broader definitions of bipolar disorder than DSM-IV-defined bipolar disorder. Subdiagnostic features of bipolar disorder are relatively common in primary care patients with unipolar depression and are associated with a more morbid course of illness. Future classifications of recurrent depression should include dimensional measures of bipolar symptoms.

\section{Declaration of interest}

D.J.S. has received honoraria for speaking at educational meetings organised by AstraZeneca and Lilly.
Bipolar disorder is a complex mood disorder that can be viewed as a spectrum condition. ${ }^{1,2}$ A growing body of research suggests that it is underrecognised in clinical practice and often misdiagnosed as recurrent major depressive disorder. ${ }^{3-8}$ Estimates of the mean delay between first onset of mood symptoms and receiving a correct bipolar diagnosis are in the region of 10 years. $^{9-11}$ Certain subgroups of individuals with depression, such as those with early-onset depression ${ }^{12,13}$ and those with severe or treatment-refractory depression, ${ }^{14}$ appear to have the highest rates of unrecognised bipolar disorder. However, many of the studies that have identified underdiagnosis of bipolar disorder have been carried out in secondary or tertiary care settings and there have as yet been no studies in the UK assessing the likely prevalence of unrecognised bipolar disorder in primary care patients who have a working diagnosis of unipolar depression.

This study had three main aims. First, to estimate the proportion of primary care patients with a working diagnosis of unipolar depression who satisfy DSM-IV ${ }^{15}$ diagnostic criteria for bipolar disorder (either bipolar I disorder, bipolar II disorder or bipolar disorder not otherwise specified). Second, to test the potential usefulness of two screening instruments for bipolar disorder - the Hypomania Checklist (HCL-32) ${ }^{16}$ and Bipolar Spectrum Diagnostic Scale $(B S D S)^{17}$ - within a primary care sample. Third, to assess whether those participants with major depressive disorder who screen false positive for bipolar disorder on the HCL-32 or BSDS questionnaires (that is, participants with major depressive disorder and with a history of subdiagnostic manic symptoms) differ from participants with major depressive disorder but with no or only minimal history of manic symptoms

†See editorial, pp. 3-4, this issue. in terms of their clinical course, psychosocial functioning and quality of life.

\section{Method}

\section{Recruitment}

This study was approved by the South East Wales Research Ethics Committee, Cardiff and Vale University Health Board, and Cardiff, Merthyr Tydfil and Newport Local Health Boards (primary care local health boards). Our aim was to collect detailed diagnostic, clinical, psychosocial functioning and quality of life data on a representative sample of individuals from primary care who had a current working diagnosis of unipolar depression. In order to sample people from a range of socioeconomic backgrounds, we invited 45 general practices in three local health boards in South Wales to take part in this study (27 practices from Cardiff Local Health Board, 9 from Merthyr Tydfil Local Health Board and 9 from Newport Local Health Board). Eleven practices agreed to take part (seven from Cardiff, three from Merthyr and one from Newport). From their patient databases, practice managers generated lists of individuals who had at least one of eight possible codes for depression recorded by their general practitioner (GP) within the past 5 years. These codes were: 'recurrent depressive disorder'; 'depressive episode'; 'depressive disorder NEC (not elsewhere specified)'; 'recurrent major depressive episode'; 'single major depressive episode'; 'neurotic (reactive) depression'; 'depressed'; and 'depression NOS (not otherwise specified)'.

When large numbers of potentially eligible participants were identified within a single practice, a maximum of 400 were selected using computer-generated random number lists. In total, 3117 patients were identified as potentially eligible from the 
11 practices and all were invited to take part in this study. The invitation sheet about the study was sent to individuals by practice managers on behalf of the research team and included the HCL-32 and BSDS questionnaires. Invited participants were asked to return the completed questionnaires if they were interested in taking part in the study. A reminder invitation pack was sent by practice managers to those who had not replied after 8 weeks.

The recruitment process is outlined in Fig. 1. In total, 576 individuals (18.5\% of those invited) volunteered to take part and returned completed HCL-32 and BSDS questionnaires. In order to compare volunteers and non-volunteers, each practice manager used computer-generated random number lists to select a small sample of 55 volunteers and 55 non-volunteers (5 volunteers and 5 non-volunteers from each practice) and performed a case-file review of age, gender, time since first diagnosis of depression, whether currently taking antidepressant medication, whether taking other medication, and whether diagnosed with a comorbid medical disorder (Table 1).

The 576 participants who returned HCL-32 and BSDS questionnaires were divided into two groups: a group of 'high scorers' (defined as scoring either 14 or more on the HCL-32 or 13 or more on the BSDS; $n=411$ ) and a group of 'low scorers' (defined as scoring less than 14 on the HCL-32 and less than 13 on the BSDS; $n=165$ ) (Fig. 1). This approach is outlined in the recruitment flow chart (Fig. 1). The choice of these thresholds was informed by several previous studies that suggested that 14 or more on the HCL-32 and 13 or more on the BSDS represented reasonable cut-offs on each questionnaire for reliably distinguishing between bipolar disorder and major depressive disorder. ${ }^{16-20}$

From the 576 volunteers, all of the 'low scorers' group $(n=165)$ and $50 \%$ of the 'high scorers' group (selected using computer-generated random number lists; $n=205)$ were invited to take part in a comprehensive diagnostic and clinical assessment. In total, $154(41.6 \%)$ of these 370 eligible participants were assessed at interview. To test the representativeness of this sample, the 154 participants who were interviewed were compared with the 216 participants who declined to be interviewed (Table 2).

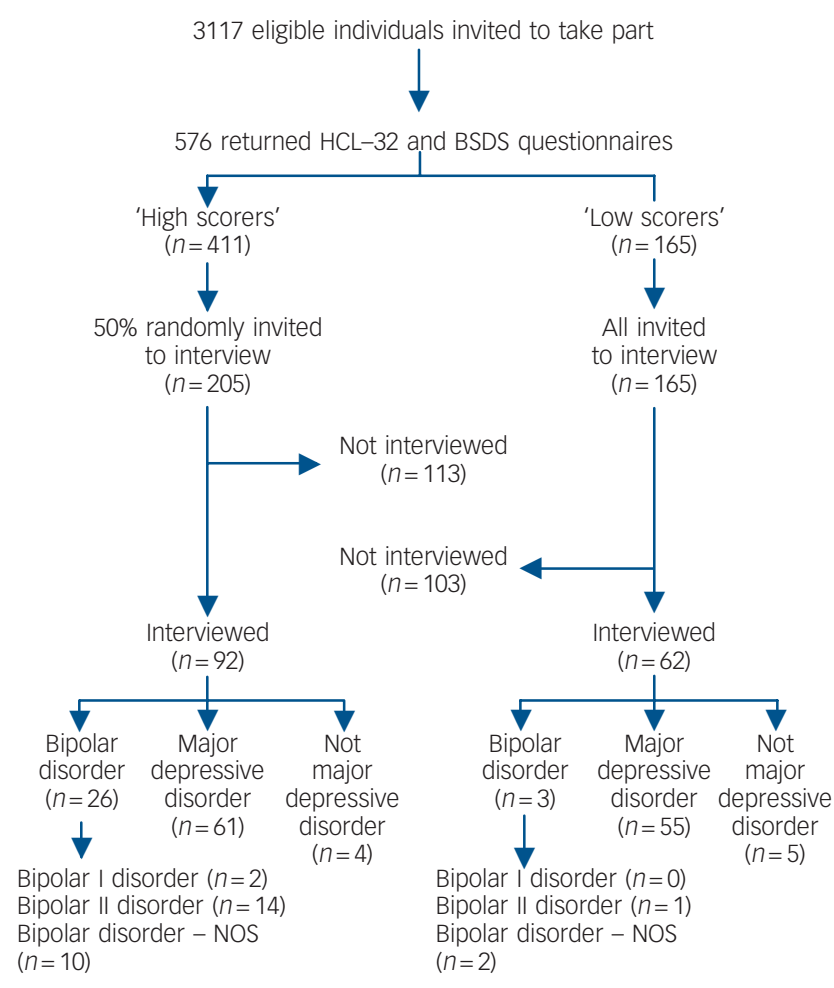

Fig. 1 Recruitment flow chart.

'High scorers' were defined as having either a score of 14 or more on the Hypomania Checklist-32 (HCL-32) or 13 or more on the Bipolar Spectrum Diagnostic Scale (BSDS). 'Low scorers' were defined as having a score of less than 14 on the HCL-32 and less than 13 on the BSDS. NOS, not otherwise specified.

\section{Assessments}

The comprehensive diagnostic and clinical assessment was carried out by a research psychologist and included: the Mini International Neuropsychiatric Interview (MINI) to obtain DSM-IV diagnoses; ${ }^{21}$ a structured assessment of sociodemographics, medical history, current medication and family

\begin{tabular}{|c|c|c|c|}
\hline & Volunteers $(n=55)$ & Non-volunteers $(n=55)$ & $P$ \\
\hline Age, years: mean (s.d.) & $42.8(13.0)$ & $44.4(11.1)$ & $0.49^{\mathrm{a}}$ \\
\hline Gender ratio, male:female & $17: 38$ & 18:37 & $0.84^{\mathrm{b}}$ \\
\hline Time since first diagnosis, months: median (range) & $60(4-492)$ & $48(3-540)$ & $0.51^{\mathrm{c}}$ \\
\hline Currently taking antidepressants, $n$ (\%) & $32(58.2)$ & $18(32.7)$ & $0.01^{\mathrm{b}}$ \\
\hline Currently taking other medications, $n$ (\%) & 35 (63.6) & $27(49.1)$ & $0.12^{b}$ \\
\hline At least 1 comorbid medical disorder, $n$ (\%) & $28(50.9)$ & $22(40.0)$ & $0.29^{b}$ \\
\hline $\begin{array}{l}\text { a. Independent } t \text {-test. } \\
\text { b. } \chi^{2} \text {-test. } \\
\text { c. Mann-Whitney U-test. }\end{array}$ & & & \\
\hline
\end{tabular}

Table 2 Comparison of interviewees $v$. those who were not interviewed

\begin{tabular}{|c|c|c|c|}
\hline & Interviewed $(n=154)$ & Not interviewed $(n=216)$ & $P$ \\
\hline Age, years: mean (s.d.) & $44.0(12.5)$ & $40.9(13.7)$ & $0.03^{\mathrm{a}}$ \\
\hline Male, $n(\%)$ & $53(34.4)$ & $58(26.9)$ & $0.12^{b}$ \\
\hline Ethnicity, White: $n(\%)$ & $118(76.6)$ & $155(71.8)$ & $0.17^{b}$ \\
\hline Hypomania Checklist-32 score, mean (s.d.) & $12.83(7.14)$ & $14.09(7.46)$ & $0.10^{\mathrm{a}}$ \\
\hline Bipolar Spectrum Diagnostic Scale score, mean (s.d.) & $11.77(6.14)$ & $12.66(6.45)$ & $0.19^{\mathrm{a}}$ \\
\hline $\begin{array}{l}\text { a. Independent } t \text {-test. } \\
\text { b. } \chi^{2} \text {-test. }\end{array}$ & & & \\
\hline
\end{tabular}


history of psychiatric disorder; the Montgomery-Åsberg Depression Rating Scale (MADRS) ${ }^{22}$ and the Young Mania Rating Scale (YMRS) ${ }^{23}$ to assess levels of current depressive and manic symptoms respectively; the Massachusetts General Hospital (MGH) assessment of treatment resistance to antidepressants; ${ }^{24}$ the Global Assessment of Functioning (GAF) scale; ${ }^{25}$ the Functional Assessment Screening Tool (FAST) ${ }^{26}$ and the World Health Organization Quality of Life Instrument (WHOQOL-Bref). ${ }^{27}$

\section{Two-phase sampling analysis}

The prevalence of undiagnosed bipolar disorder observed in the data was estimated using Dunn's two-phase sampling strategy (Fig.1). ${ }^{28}$ This approach applies to survey designs where the population of interest is screened and then split into multiple arms based on screening scores. Differential proportions are invited to further testing/interview to ensure broadly equal numbers of participants in each arm. Point estimates of the prevalence along with $95 \%$ confidence intervals were calculated using weighted logistic regression.

Three increasingly conservative sets of assumptions were made about participants who did not respond either to the initial questionnaire invitation or subsequently to the invitation to interview in order to provide robust estimates of the proportion of undiagnosed bipolar in this population. First, least conservatively, we made the assumption that all of those who were not interviewed and all of those who did not return their questionnaires would have responded similarly to those who were interviewed (Fig. 1). Here we assume that the 92 high scorers who were interviewed represent all of the 411 high scorers (a sampling weight of 4.4) and that the 62 low scorers who were interviewed represent all 165 low scorers (a sampling weight of 2.7). This provides the least conservative estimate of the prevalence of undiagnosed bipolar disorder in this sample. Second, more conservatively, we assumed that all of those who were not interviewed did not have bipolar disorder and that the 576 participants who returned their questionnaires were representative of the 3117 individuals with unipolar depression initially approached. This means that the 205 high scorers who were invited to interview (comprising 113 high-scoring participants not interviewed who are assumed not to have bipolar disorder and 92 who were interviewed) represent the 411 high scorers. This calculation, with a weighting of 2 for the 205 high scorers and 1 for the 165 low scorers, gives an intermediate estimate of undiagnosed bipolar disorder. Finally, the most conservative estimate makes the assumption that all of those who dropped out at either stage (either through failing to return the initial screening questionnaire or not having been interviewed when invited) did not have bipolar disorder. The 205 high scorers invited to interview represent the 411 high scorers and the 165 low scorers represent everyone else (the weighting for the 205 high scorers is 2.0 and the weights for the 165 low scorers is 16.4 ).

\section{Receiver Operating Characteristic curve analyses}

To assess the potential usefulness of the HCL-32 and BSDS questionnaires in this sample, we conducted receiver operating characteristic (ROC) curve analyses between participants with DSM-IV-defined bipolar disorder and major depressive disorder. Optimal thresholds were based on maximising the sum of the sensitivity and specificity.

\section{Comparing participants with major depressive disorder with and without a history of manic symptoms}

To assess whether participants with major depressive disorder with a history of subdiagnostic manic symptoms differed from those participants with no or minimal history of manic symptoms, we divided participants with major depressive disorder into two groups: one group of 'false positives' who scored above the optimal calculated threshold for bipolar disorder on either the HCL-32 or the BSDS (the major depressive disorder (MDD)-manic symptoms group) and another group who were 'true negatives' in that they scored below the threshold for bipolar disorder on both screening instruments (called the MDD group). For each questionnaire, these groups were then compared with each other on a range of demographic, clinical, psychosocial functioning and quality of life measures. We used SPSS for Windows, version 11.5), to conduct chi-squared tests for dichotomous variables, the Mann-Whitney $U$-test for non-parametric data and independent $t$-tests for parametric data. Given the relatively large number of comparisons between groups, statistical significance was set at 0.01 .

\section{Results}

\section{Sample representativeness}

Volunteers for the study who returned their questionnaires and those who did not return their questionnaires were generally well matched apart from whether they were currently taking antidepressants $(58.2 \%$ of volunteers $v .32 .7 \%$ of non-volunteers; Table 1). There were no differences between participants who were and were not subsequently interviewed in terms of gender ratio, ethnicity and mean HCL-32 and BSDS scores, although the interviewed group were younger (mean age 44.0 years $v$. 40.9 years, $P=0.03$ ) (Table 2).

\section{Estimates of previously unrecognised bipolar disorder}

From the 154 participants interviewed, 9 (5.8\%) had not previously experienced an episode of major depression as defined by DSM-IV. In total, $116(75.3 \%)$ had a history of DSM-IV major depressive disorder and 29 (18.8\%) satisfied DSM-IV criteria for bipolar disorder (including bipolar I disorder, $n=2$; bipolar II disorder, $n=15$; and bipolar disorder not otherwise specified, $n=12$ ) (Fig. 1).

Following the two-phase sampling methodology proposed by Dunn et $a{ }^{28}$ three increasingly conservative estimates of previously unrecognised bipolar disorder in this sample were possible. The least conservative estimate, which makes the assumption that all of those who were not interviewed and all of those who did not return their questionnaires would have responded similarly to those who were interviewed, is $21.6 \%$ (95\% CI 18.4-25.1). A more conservative estimate assumes that all of those who were not interviewed did not have bipolar disorder and that the 576 participants who returned their questionnaires were representative of the 3117 individuals with unipolar depression initially approached, and gives an estimate of previously undiagnosed bipolar disorder of 9.6\% (95\% CI 7.4-12.3). Finally, the most conservative estimate makes the assumption that all of those who dropped out at either stage (either through failing to return the initial screening questionnaire or not having been interviewed when invited) did not have bipolar disorder. This gives an estimate of 3.3\% (95\% CI 2.7-3.9).

\section{HCL-32 and BSDS screening questionnaires in primary care}

In this sample, both the HCL-32 and BSDS could distinguish between bipolar disorder and major depressive disorder with reasonable precision (the area under the curve was 0.81 for the HCL-32 and 0.71 for the BSDS) (Figs 2 and 3). However, even though the optimum thresholds of 18 or more for the HCL-32 


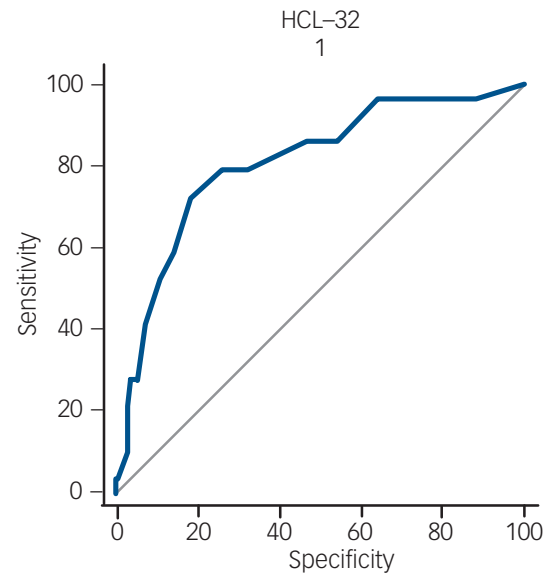

\begin{tabular}{|lcccc|}
\hline $\begin{array}{l}\text { HCL-32 } \\
\text { Score }\end{array}$ & Sensitivity & Specificity & $\begin{array}{c}\text { Positive } \\
\text { predictive } \\
\text { values }\end{array}$ & $\begin{array}{c}\text { Negative } \\
\text { predictive } \\
\text { values }\end{array}$ \\
\hline$>15$ & 79.31 & 74.14 & 43.4 & 93.5 \\
\hline$>16$ & 75.86 & 77.59 & 45.8 & 92.8 \\
\hline$>17^{\text {a }}$ & 72.41 & 81.90 & 50.0 & 92.2 \\
\hline$>18$ & 58.62 & 86.21 & 51.5 & 89.3 \\
\hline$>19$ & 51.72 & 89.66 & 55.6 & 88.1 \\
\hline a. Optimal threshold 18 or more. & & & \\
\hline
\end{tabular}

Fig. 2 Receiver operating characteristic curve analysis for the Hypomania Checklist-32 (HCL-32): bipolar disorder $(n=29)$ v. major depressive disorder $(n=116)$.

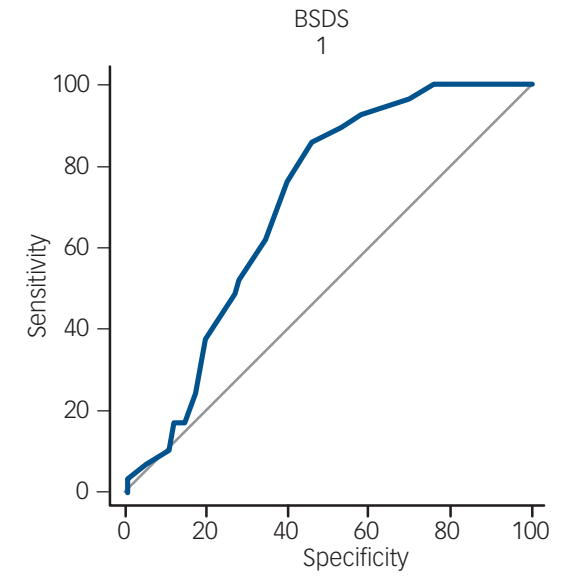

\begin{tabular}{|lcccc|}
\hline $\begin{array}{l}\text { BSDS } \\
\text { Score }\end{array}$ & Sensitivity & Specificity & $\begin{array}{c}\text { Positive } \\
\text { predictive } \\
\text { values }\end{array}$ & $\begin{array}{c}\text { Negative } \\
\text { predictive } \\
\text { values }\end{array}$ \\
\hline$>9$ & 93.10 & 41.38 & 28.4 & 96.0 \\
\hline$>10$ & 89.66 & 46.55 & 29.5 & 94.7 \\
\hline$>11^{a}$ & 86.21 & 54.31 & 32.1 & 94.0 \\
\hline$>12$ & 75.86 & 60.34 & 32.4 & 90.9 \\
\hline$>13$ & 62.07 & 65.52 & 31.0 & 87.4 \\
\hline & & & \\
\hline
\end{tabular}

Fig. 3 Receiver operating characteristic curve analysis for the Bipolar Spectrum Diagnostic Scale (BSDS): bipolar disorder $(n=29)$ V. major depressive disorder $(n=116)$. and 12 or more for the BSDS had reasonable sensitivities and specificities, the positive predictive values for both questionnaires were quite low (50.0\% for the HCL-32 and $32.1 \%$ for the BSDS).

\section{Subthreshold manic symptoms in major depressive disorder}

The sample of 116 participants with major depressive disorder were divided into two groups according to their scores on each of the questionnaires: a MDD-manic symptoms group $(n=60)$ and an MDD group $(n=56)$. These groups were compared with each other on a range of clinical, psychosocial functioning and quality of life measures (Table 3; see online Table DS1 for a more detailed version of this table.). For both the HCL-32 threshold definition of major depressive disorder with manic symptoms (a score of 18 or more) and the BSDS definition (a score of 12 or more) there were no significant differences between the two groups on several demographic (gender, ethnicity, employment status, premorbid IQ assessed with the National Adult Reading Test) ${ }^{29}$ and clinical variables (whether ever admitted to hospital for depression, antidepressant treatment resistance, whether currently taking psychotropic medication and family history of depression and bipolar disorder) (Table 3). However, for the HCL-32 definition, the MDD-manic symptoms group were significantly younger, had an earlier age at onset of depression, reported more current depressive symptoms, had more frequent episodes of depression and were more likely to have a comorbid history of alcohol misuse and alcohol dependence. This group also had significantly poorer functioning rated by the GAF, more difficulties with relationships according to the FAST questionnaire and lower scores on the overall score for the WHOQOL-Bref quality of life measure (including lower scores within the 'psychological' and 'environment' subsections).

Similarly, the MDD-manic symptoms group defined by the BSDS threshold were significantly younger, had more current depressive symptoms, were more likely to have a history of chronic depression, had more lifetime comorbid panic, alcohol misuse and alcohol dependence disorders and had worse current functioning measured by the GAF. They were significantly more impaired in all of the FAST psychosocial functioning sections (including 'autonomy', 'occupational', 'cognitive', 'finances', 'relationships' and 'leisure time') and all of the WHOQOl-Bref quality of life sections ('physical health', 'psychological', social relationships' and 'environment') (Table 3 and online Table DS1).

Overall, these findings suggest that the MDD-manic symptoms group (whether defined by the HCL-32 or the BSDS) had a pattern of depressive illness that was more severe and that was associated with both poorer psychosocial functioning and worse quality of life than participants with major depressive disorder with no or little history of subthreshold manic symptoms.

\section{Discussion}

There are three key findings from this study. First, unrecognised DSM-IV bipolar disorder may be relatively common in primary care patients with a working diagnosis of unipolar depression. Our most conservative estimate was $3.3 \%$ and our least conservative estimate was $21.6 \%$. Second, the HCL-32 and BSDS screening questionnaires (when used in primary care settings) may have limited utility in terms of detecting DSM-IV-defined bipolar 


\begin{tabular}{|c|c|c|c|c|c|c|}
\hline & \multicolumn{3}{|c|}{ Hypomania Checklist-32 } & \multicolumn{3}{|c|}{ Bipolar Spectrum Diagnostic Scale } \\
\hline & $\begin{array}{c}\text { MDD-manic } \\
\text { symptoms group } \\
\text { (score } 18 \text { or more) } \\
(n=42)\end{array}$ & $\begin{array}{l}\text { MDD group } \\
\text { (score } 17 \text { or less) } \\
(n=103)\end{array}$ & $P$ & $\begin{array}{c}\text { MDD-manic } \\
\text { symptoms group } \\
\text { (score } 12 \text { or more) } \\
(n=78)\end{array}$ & $\begin{array}{l}\text { MDD group } \\
\text { (score } 11 \text { or less) } \\
(n=67)\end{array}$ & $P$ \\
\hline Age, years: mean (s.d.) & $38.7(9.5)$ & $46.7(12.8)$ & $0.0004^{\mathrm{a}}$ & $41.7(11.4)$ & $47.6(12.9)$ & $0.004^{\mathrm{a}}$ \\
\hline Male, $n(\%)$ & $19(45)$ & $27(26)$ & $0.03^{b}$ & $29(37)$ & $17(25)$ & $0.13^{\mathrm{b}}$ \\
\hline Ethnicity, White: $n$ (\%) & $41(98)$ & $100(97)$ & $0.86^{\mathrm{b}}$ & $76(97)$ & $65(97)$ & $0.88^{b}$ \\
\hline Currently employed, $n$ (\%) & $19(45)$ & $41(40)$ & $0.55^{\mathrm{b}}$ & $32(41)$ & $28(42)$ & $0.93^{\mathrm{b}}$ \\
\hline Premorbid IQ (NART), median (range) & $35(4-54)$ & $31(4-49)$ & $0.14^{c}$ & $32(4-54)$ & $34(4-48)$ & $0.58^{c}$ \\
\hline Age at onset of depression, years: mean (s.d.) & $20.6(8.1)$ & $26.6(14.2)$ & $0.01^{a}$ & $22.7(12.0)$ & $27.3(13.7)$ & $0.03^{\mathrm{a}}$ \\
\hline MADRS, median (range) & $17.5(0-35)$ & $10.0(0-49)$ & $0.01^{\mathrm{c}}$ & $17(0-40)$ & $7(0-49)$ & $0.00004^{c}$ \\
\hline YMRS, median (range) & $0(0-5)$ & $0(0-5)$ & $0.04^{c}$ & $0(0-5)$ & $0(0-5)$ & $0.03^{c}$ \\
\hline Chronic depression, $n$ (\%) & $20(48)$ & $58(56)$ & $0.34^{b}$ & $34(44)$ & $44(66)$ & $0.008^{\mathrm{b}}$ \\
\hline Episodes of depression, ${ }^{d}$ median (range) & $6(3-40)$ & $3(1-30)$ & $0.003^{c}$ & $6(1-40)$ & $3(1-30)$ & $0.02^{c}$ \\
\hline Ever admitted, $n$ (\%) & $4(10)$ & $12(12)$ & $0.71^{b}$ & $8(10)$ & $8(12)$ & $0.75^{\mathrm{b}}$ \\
\hline MGH score, median (range) & $1.5(0-8)$ & $1.5(0-8)$ & $0.97^{\mathrm{C}}$ & $1.5(0-10)$ & $1.5(0-6)$ & $0.13^{c}$ \\
\hline On psychotropic medication, $n$ (\%) & $35(83)$ & $79(77)$ & $0.38^{\mathrm{b}}$ & $62(80)$ & $52(78)$ & $0.78^{\mathrm{b}}$ \\
\hline First-degree relative with depression, $n$ (\%) & $23(55)$ & $47(46)$ & $0.32^{b}$ & $40(51)$ & $30(45)$ & $0.44^{\mathrm{b}}$ \\
\hline First-degree relative with bipolar disorder, $n$ (\%) & $4(10)$ & $3(3)$ & $0.09^{b}$ & $3(4)$ & $4(6)$ & $0.55^{\mathrm{b}}$ \\
\hline Comorbid panic disorder, $n(\%)$ & $18(31)$ & $25(24)$ & $0.41^{b}$ & $27(35)$ & $11(16)$ & $0.01^{\mathrm{b}}$ \\
\hline Comorbid alcohol misuse, $n(\%)$ & $23(55)$ & $18(18)$ & $0.00001^{b}$ & 29 (37) & $12(18)$ & $0.01^{\mathrm{b}}$ \\
\hline Comorbid alcohol dependence, $n$ (\%) & $17(41)$ & $7(7)$ & $0.00001^{b}$ & $19(24)$ & $5(8)$ & $0.006^{b}$ \\
\hline GAF score, mean (s.d.) & $63.4(14)$ & $69.0(12.4)$ & $0.001^{a}$ & $64.1(12.3)$ & $71.2(13.1)$ & $0.001^{a}$ \\
\hline FAST: total score, median (range) & $31(1-70)$ & $24(0-62)$ & $0.02^{c}$ & $31(1-70)$ & $18(0-57)$ & $0.0001^{c}$ \\
\hline WHOQOL-Bref, total score: median (range) & $45.5(19-69)$ & $51(29-73)$ & $0.007^{\mathrm{C}}$ & $47(19-68)$ & $55(29-73)$ & $0.00002^{c}$ \\
\hline \multicolumn{7}{|c|}{$\begin{array}{l}\text { MDD, major depressive disorder; NART, National Adult Reading Test; MADRS, Montgomery-Åsberg Depression Rating Scale; YMRS, Young Mania Rating Scale; MGH, Massachusetts } \\
\text { General Hospital; GAF, Global Assessment of Functioning scale; FAST, Functional Assessment Screening Tool; WHOQOL-Bref, World Health Organization Quality of Life Instrument. } \\
\text { a. Independent } t \text {-test. } \\
\text { b. } \chi^{2} \text {-test. } \\
\text { c. Mann-Whitney U-test. } \\
\text { d. For episodes of depression: sample size } n=78 \text {. }\end{array}$} \\
\hline
\end{tabular}

disorder, although it is possible that they may be useful for broader definitions of bipolar disorder. Third, a history of manic symptoms in primary care patients with DSM-IV major depressive disorder is relatively common and is associated with a more morbid course of illness, worse psychosocial functioning and poorer quality of life.

\section{Underdiagnosis of bipolar disorder in primary care}

Our first finding, that unrecognised bipolar disorder could be diagnosed in at least $3.3 \%$ and at most $21.6 \%$ of our sample of primary care patients with unipolar depression, is in keeping with several studies that have found that bipolar disorder is often misdiagnosed as major depressive disorder. ${ }^{1,3-7,30}$ To our knowledge, our study is the first to investigate this issue in a sample of primary care patients within the UK. The three estimates of prevalence of previously undiagnosed bipolar disorder we obtained (21.6, 9.6 and 3.3\%) were each based on different assumptions. The smallest proportion of $3.3 \%$ was based on the strictest assumption that all individuals who dropped out in this study did not have bipolar disorder. This seems unlikely and therefore provides an extreme lower bound for the proportion of people with undiagnosed bipolar disorder in this population. Even under the assumption that all of those who were invited to interview but not interviewed did not have bipolar disorder (and that the 576 responders were representative of the 3117 approached) the proportion of people with undiagnosed bipolar disorder was as high as $9.6 \%$.
Although our findings will require replication, we suggest that at least 1 in 30 patients in primary care with a working diagnosis of unipolar depression may in fact have unrecognised bipolar disorder. Although challenging, this is potentially a finding with considerable implications for they way in which GPs approach the diagnosis and treatment of their patients with depression, ${ }^{31}$ especially when we consider how commonly antidepressants are prescribed in primary care and the potential for harm when antidepressants are used as monotherapy for bipolar disorder. Although there have as yet been no empirical studies of antidepressant therapy for subthreshold bipolar disorder, antidepressants are now known to be of only limited benefit in the treatment of DSM-IV bipolar depression ${ }^{32,33}$ and, at least for a proportion of individual's with a DSM-IV bipolar diagnosis, they may be unhelpful by causing suicidal behaviour, treatment resistance and more frequent cycling of mood episodes. ${ }^{34}$

Greater recognition of bipolar disorders in primary care will also have important resource implications for the way in which primary and secondary care services work together to manage individuals with depression.

\section{Use of the HCL-32 and BSDS screening instruments in primary care}

Although the HCL-32 and BSDS questionnaires had relatively low positive predictive values for detecting DSM-IV bipolar disorder (50.0\% for the HCL-32 and $32.1 \%$ for the BSDS), it should be noted that there is now a consensus that the definition of bipolar 
disorder (particularly bipolar II disorder) within DSM-IV is overly restrictive and, further, that these questionnaires were designed to identify broadly defined hypomania. The American Psychiatric Association's proposed draft of DSM-5 (www.dsm5.org) recommends a broadening of the diagnostic criteria for hypomania to include increased activity/energy as an additional core (or 'gate') symptom and the removal of antidepressant-associated hypomania as an exclusion criterion for hypomania. It is therefore likely that the gold-standard (DSM-IV) definition we have used in this study is too restrictive and it is possible that the HCL-32 and BSDS may have had improved positive predictive values had we used broader diagnostic criteria for hypomania. Further work on these instruments is important because there is currently a vogue for the unsupervised use of these questionnaires by patients (for example, via the internet) and GPs are increasingly faced with having to explain to people why scoring highly on such questionnaires may or may not be diagnostic of bipolar disorder.

\section{The broad clinical spectrum of bipolar disorder}

Major depressive disorder may be a heterogeneous diagnostic grouping that contains a substantial proportion of individuals who have subtle (but clinically relevant) bipolar features that fall just below the DSM-IV diagnostic threshold for bipolar disorder. ${ }^{35}$ Several recent epidemiological studies support the view that mild or 'subthreshold' features of bipolar disorder in people with a diagnosis of major depression are relatively common and clinically important.

In the latest wave of the Munich Early Developmental Stages of Psychopathology (EDSP) Study, 202 participants (41.4\%) out of 488 identified with DSM-IV major depressive disorder fulfilled diagnostic criteria for 'subthreshold bipolar disorder. ${ }^{36}$ The criteria for subthreshold bipolar disorder in this study were: major depression, plus hypomania defined as 'at least 4 days of either elated or expansive mood that caused problems or that was noticed by others (plus $<3$ other manic symptoms), or unusually irritable mood plus at least 3 other manic symptoms (not observed by others)'. These participants with subthreshold bipolar disorder differed from the remaining participants with major depressive disorder on a number of clinical variables. As a group, they had a significantly higher rate of family history of mania, higher rates of nicotine dependence and alcohol use disorders, higher rates of panic disorder, a tendency to more frequent criminality and a greater prospective risk of converting to DSM-IV bipolar disorder. This suggests that they represent a clinically important bipolar subgroup that is intermediate between DSM-IV bipolar disorder and DSM-IV major depressive disorder.

Similar findings were observed in the Zurich Longitudinal Study that used two definitions of hypomania that were less restrictive than the DSM-IV definition of hypomania (the so-called 'Strict Zurich' and 'Broad Zurich' criteria). ${ }^{37}$ Under DSM-IV criteria for hypomania, $7.4 \%$ of the Zurich cohort fulfilled criteria for bipolar II disorder but using the Strict Zurich criteria this figure rose to $23.7 \%$ and according to the Broad Zurich criteria the figure was $49.2 \% .^{37}$

In the US National Comorbidity Replication Study, Merikangas and colleagues defined subthreshold bipolar disorder as hypomania without a history of major depression or with fewer symptoms than required for DSM-IV hypomania. ${ }^{38}$ They found lifetime prevalence rates of $1.0 \%$ for bipolar I disorder, $1.1 \%$ for bipolar II disorder and 2.4\% for subthreshold bipolar disorder. Participants with subthreshold bipolar disorder were not clinically benign. They had frequent psychiatric comorbidity, moderate to severe clinical severity, significant role impairment and only rarely had been prescribed mood stabilising medication. Furthermore, almost $40 \%$ of study participants with a history of major depressive disorder also had a history of subthreshold hypomania and these participants had a younger age at onset, more episodes of depression and higher rates of comorbidity. ${ }^{39}$

Our finding that people with major depressive disorder from primary care with a history of subdiagnostic manic symptoms were different from individuals with major depressive disorder with no or minimal history of manic symptoms on some clinical features and on several measures of both psychosocial functioning and quality of life is consistent with the findings in the Zurich longitudinal study, ${ }^{37}$ the Munich EDSP Study ${ }^{36}$ and the National Comorbidity Replication Study. ${ }^{38,39}$ Taken together, these studies suggest that low-grade, subdiagnostic levels of manic symptoms in people with major depressive disorder are clinically important and are associated with a more morbid illness course, poorer functioning and worse quality of life.

Although in this study we have used DSM-IV definitions of bipolar disorder as the gold-standard diagnoses, as noted above, there are limitations with a threshold approach to diagnosing bipolar disorder. One possible interpretation of our findings is that they provide support for a dimensional view of bipolar disorder that goes beyond the use of formal (and somewhat arbitrary) DSM-IV categories and thresholds. It may be that ICD-11 and DSM-5 should incorporate the measurement of dimensions of bipolar symptoms into the diagnostic criteria for both recurrent major depressive disorder and bipolar disorder.

A further suggestion is that assessing manic symptoms should become part of the routine diagnostic assessment of all individuals presenting with depression and that these assessments should guide treatment, for example, with regard to antidepressants. If we extrapolate from studies of DSM-IV bipolar depression, it is possible that many people with major depressive disorder who also have subdiagnostic bipolar features will not benefit from antidepressant therapy and may even do worse in the longer term because antidepressants could induce a course of depressive disorder with more frequent relapses and worsening mood instability. This is potentially a very large group of people and it is important that future research is less constrained by strict DSM-IV diagnoses and instead tries to evaluate the likely usefulness (or harm) caused by antidepressants in those people with depression who have subthreshold or mild features of bipolar disorder.

\section{Strengths of this study}

This is the first large-scale, systematic assessment of bipolar features in individuals with depression within a UK primary care setting. We were careful to recruit from several practices with a spread of participants from different social and economic backgrounds and we carried out formal diagnostic assessments on a representative subsample. As noted earlier, the two-stage methodological approach recommended by Dunn and colleagues $^{28}$ allowed us to obtain three increasingly conservative estimates of the likely prevalence of unrecognised bipolar disorder within our sample.

\section{Limitations of this study}

There are several limitations in this study. Only $18.5 \%$ of the 3117 people we invited to take part volunteered and returned their questionnaires. In general, volunteers for this study were similar to non-volunteers in terms of age, gender, time since diagnosis, medication use and medical comorbidity (Table 1). However, volunteers were significantly more likely to be taking 
antidepressant medication than non-volunteers. This could have several implications. It may suggest that those who volunteered had a more severe form of depressive illness; that they were more likely to report low-grade manic symptoms which were being caused by antidepressants; that they had depression which was treated by antidepressants and they were therefore more motivated to take part in a research study; or even that they were more interested in taking part because their current antidepressant treatment was ineffective. It is also possible, however, that individuals with bipolar disorder were overrepresented in the group who did not volunteer because they were resistant to the possibility of receiving this diagnosis during the study.

Similarly, from the group of participants who were invited to interview, those who were interviewed were similar to those who were not interviewed in terms of gender, ethnicity and mean HCL-32 and BSDS scores but were significantly older as a group. This age difference (although relatively small at only 4.1 years) could make it more likely that the interviewed group would contain more individuals with bipolar disorder.

\section{Implications}

In summary, our findings suggest that a significant minority of primary care patients with a working diagnosis of unipolar depression (between 3.3 and 21.6\%) may have an undiagnosed DSM-IV bipolar disorder. Across the UK, this could represent a large number of individuals. Given the likelihood that many of these individuals are receiving antidepressants as monotherapy (which may be at best unhelpful and at worst harmful), it will be important that GPs are supported in developing strategies to ensure that their patients with depression receive the correct diagnosis with regard to the possibility of a primary bipolar illness. We also found that the HCL-32 and BSDS screening questionnaires had relatively low positive predictive values for DSM-IV bipolar disorder but it is possible that they could be useful for identifying broader definitions of bipolar disorder. Finally, in keeping with several other studies from around the world, we found that subthreshold features of bipolar disorder were relatively common in individuals with unipolar depression (even in primary care) and were associated with a more morbid course of illness and greater psychosocial and quality of life impairments. These findings have important implications for the classification, assessment and treatment of large numbers of people with depression managed in both primary and secondary care settings.

\footnotetext{
Daniel J. Smith, MD, MRCPsych, Emily Griffiths, BSC, Department of Psychological
Medicine and Neurology, Cardiff University School of Medicine, University Hospital of Medicine and Neurology, Cardiff University School of Medicine, University Hospital of
Wales, Cardiff; Mark Kelly, PhD, Kerry Hood, PhD, Department of Primary Care and Public Health, Cardiff University School of Medicine, University Hospital of Wales, Cardiff; Nick Craddock, PhD, FRCPsych, Department of Psychological Medicine and Neurology, Cardiff University School of Medicine, University Hospital of Wales, Cardiff; Sharon A. Simpson, PhD, Department of Primary Care and Public Health, Cardiff

Correspondence: Daniel J. Smith, MD, MRCPsych, Department of Psychologica Medicine and Neurology, Cardiff University School of Medicine, Monmouth House (second floor), University Hospital of Wales, Heath Park, Cardiff, CF14 4DW, UK. Email: smithdj3@cardiff.ac.uk

First received 29 Jun 2010, final revision 31 Aug 2010, accepted 3 Nov 2010
}

\section{Funding}

Funded by an MRC/Welsh Assembly Government Partnership Award (2008-2010). D.J.S. is funded by a Postdoctoral Fellowship from the National Institute of Health Research (NIHR).

\section{References}

1 Angst J. The bipolar spectrum. Br J Psychiatry 2007; 190: 189-91.

2 Goodwin GM, Geddes JR. What is the heartland of psychiatry? Br J Psychiatry 2007; 191: 189-91.

3 Ghaemi SN, Ko JY, Goodwin FK. Cade's Disease and beyond: misdiagnosis, antidepressant use and a proposed definition for bipolar spectrum disorder. Can J Psychiatry 2002; 47: 125-34.

4 Ghaemi SN, Sachs GS, Chiou AM, Pandurangi AK, Goodwin K. Is bipolar disorder still underdiagnosed? Are antidepressants overutilized? J Affect Disord 1999; 52: 135-44

5 Ghaemi SN, Boiman EE, Goodwin FK. Diagnosing bipolar disorder and the effect of antidepressants: a naturalistic study. J Clin Psychiatry 2000; 61: 804-8.

6 Albanese MJ, Clodfelter Jr RC, Pardo TB, Ghaemi SN. Underdiagnosis of bipolar disorder in men with substance use disorder. J Psychiatr Pract 2006; 12: $124-7$.

7 Chilakamarri JK, Filkowski MM, Ghaemi SN. Misdiagnosis of bipolar disorder in children and adolescents: a comparison with ADHD and major depressive disorder. Psychiatr Ann, in press.

8 Smith DJ, Ghaemi, N. Is underdiagnosis the main pitfall when diagnosing bipolar disorder? Yes. BMJ 2010; 340: c854.

9 Lish JD, Dime-Meenan S, Whybrow PC, Price RA, Hirschfeld RMA. The National Depressive and Manic-depressive Association (DMDA) survey of bipolar members. J Affect Disord 1994; 31: 281-94.

10 Hirschfeld RM, Lewis L, Vernik LA. Perceptions and impact of bipolar disorder: how far have we really come? Results of the National Depressive and Manic-Depressive Association 2000 survey of individuals with bipolar disorder. J Clin Psychiatry 2003; 64: 161-74.

11 Berk M, Dodd S, Callaly P, Berk L, Fitzgerald P, de Castella AR, et al. History of illness prior to a diagnosis of bipolar disorder or schizoaffective disorder. $J$ Affect Disord 2007; 103: 181-6.

12 Geller B, Zimerman B, Williams M, Bolhofner K, Craney JL. Bipolar disorder at prospective follow-up of adults who had prepubertal major depressive disorder. Am J Psychiatry 2001; 158: 125-7.

13 Smith DJ, Harrison N, Muir W, Blackwood DHR. The high prevalence of bipolar spectrum disorders in young adults with recurrent depression: toward an innovative diagnostic framework. J Affect Disord 2005; 84: 167-78.

14 Sharma $\mathrm{V}$, Khan $\mathrm{M}$, Smith A. A closer look at treatment resistant depression: is it due to a bipolar diathesis? J Affect Disord 2005; 84: 251-7.

15 American Psychiatric Association. Diagnostic and Statistical Manual of Mental Disorders (4th edn) (DSM-IV). APA, 1994.

16 Angst J, Adolfsson R, Benazzi F, Gamma A, Hantouche E, Meyer TD, et al. The HCL-32: towards a self-assessment tool for hypomanic symptoms in outpatients. J Affect Disord 200; 88: 217-33.

17 Ghaemi SN, Miller CJ, Berv DA, Klugman J, Rosenquist KJ, Pies RW. Sensitivity and specificity of a new bipolar spectrum diagnostic scale. J Affect Disord 2005; 84: 273-7.

18 Allen R, Smith DJ. Screening for bipolar disorder: strengths and limitations of currently available instruments. Prim Care Community Psychiatr 2008; 13: 47-51.

19 Carta MG, Hardoy CM, Cadeddu M, Murru A, Campus A. The accuracy of the Italian version of the Hypomania Checklist (HCL-32) for the screening of bipolar disorders and comparison with the Mood Disorder Questionnaire (MDQ) in a clinical sample. Clin Pract Epidemiol Ment Health 2006; 2: 2.

20 Vieta E, Sanchez-Moreno J, Bulbena A, Chamorro L, Ramos JL, Artal J, et al. Cross validation with the mood disorder questionnaire (MDQ) of an instrument for the detection of hypomania in Spanish: the 32 item hypomania symptom check list (HCL-32). J Affect Disord 2007; 101: 43-55.

21 Sheehan DV, Lecrubier Y, Harnett-Sheehan K, Amorim P, Janavas J, Weiller $\mathrm{E}_{\text {, et }}$ al. The Mini International Neuropsychitric Interview (MINI): the development and validation of a structured diagnostic psychiatric interview. J Clin Psychiatry 1998; 59 (suppl 20): 22-33.

22 Snaith RP, Harrop FM, Newby DA, Teale C. Grade scores of the Montgomery-Åsberg Depression and the Clinical Anxiety Scales. Br J Psychiatry 1986; 148: 599-601.

23 Young RC, Biggs JT, Ziegler VE, Meyer DA. A rating scale for mania: reliability, validity and sensitivity. Br J Psychiatry 1978; 133: 429-35.

24 Fava M. Diagnosis and definition of treatment-resistant depression. Biol Psychiatry 2003; 53: 649-59.

25 Spitzer RL, Gibbon M, Williams JBW. Global Assessment of Functioning (GAF) Scale. In Outcome Assessment in Clinical Practice (eds LI Sederer, B Dickey): 76-7. Williams and Wilkins. 
26 Rosa A, Sanchez-Moreno J, Martinez-Aran A, Salamero M, Torrent C, Reinares $\mathrm{M}$, et al. Validity and reliability of the Functioning Assessment Short Test (FAST) in bipolar disorder. Clin Pract Epidemiol Ment Health 2007; 3: 5.

27 World Health Organization. Development of the World Health Organization WHOQOL-BREF Quality of Life Assessment. Psychol Med 1998; 28: 551-8.

28 Dunn G, Pickles A, Tansella M, Vazque-Barquero JL. Two-phase epidemiological surveys in psychiatric research. Br J Psychiatry 1999; 174: 95-100

29 Nelson H, Willison J. National Adult Reading Test (2nd edn). nferNelson, 1991.

30 Angst J. Do many patients with depression suffer from bipolar disorder? Can J Psychiatry 2006; 51: 3-5.

31 Smith DJ, Thapar A, Simpson SA. Bipolar spectrum disorders in primary care: optimising diagnosis and treatment. Br J Gen Pract 2010; 60: 322-4.

32 Sachs GS, Nierenberg AA, Calabrese JR, Marangell LB, Wisniewski SR, Gyula $L F$, et al. Effectiveness of adjunctive antidepressant treatment for bipolar depression. N Engl J Med 2007; 356: 1711-22.

33 Smith DJ, Forty L, Russell E, Caesar S, Walters JT, Gordon-Smith K, et al. Subthreshold manic symptoms in recurrent major depressive disorder are a marker for poor outcome. Acta Psychiatr Scand 2009; 119: 325-9.
34 El-Mallakh RS, Karippot A, Ghaemi, SN. Antidepressants in bipolar depression. In Bipolar Depression (eds RS El-Mallakh, SN Ghaemi): 167-83. American Psychiatric Publishing, 2006.

35 Smith DJ, Ghaemi SN, Craddock, N. The broad clinical spectrum of bipolar disorder: implications for research and practice. J Psychopharmacol 2008; 22: 397-400.

36 Zimmermann P, Bruckl T, Nocon A, Pfister H, Lieb R, Wittchen $\mathrm{H}-\mathrm{U}$, et al. Heterogeneity of DSM-IV major depressive disorder as a consequence of subthreshold bipolarity. Arch Gen Psychiatry 2009; 66: 1341-52.

37 Angst J, Gamma A, Bennazzi F, Ajdacic V, Eich D, Rossler W. Toward a re-definition of subthreshold bipolarity: epidemiology and proposed criteria for bipolar-II, minor bipolar disorders and hypomania. J Affect Disord 2003: 73: $133-46$.

38 Merikangas KR, Akiskal HS, Angst J, Greenberg PE, Hirschfeld RMA, Petukhova M, et al. Lifetime and 12-month prevalence of bipolar spectrum disorder in the National Comorbidity Survey Replication. Arch Gen Psychiatry 2007; 64: 543-52.

39 Angst J, Cui L, Swendsen J, Rothen S, Cravchik A, Kessler R, et al. Major depressive disorder with subthreshold bipolarity in the national comorbidity survey replication. Am J Psychiatry 2010; 167: 1194-201. 